THE IMMUNOLOGY OF HIV

\title{
A formidable challenge
}

\section{Better understanding of HIV biology, virus-host interactions and mechanisms of an efficient immune response advance efforts for effective vaccines and immunotherapies.}

$\circlearrowleft$ in ince its discovery in 1983, human immunodeficiency virus (HIV) and the immune response to HIV infection have been studied extensively. Although attempts to develop a prophylactic or therapeutic vaccine have been largely unsuccessful, this global effort has generated valuable information on HIV biology in terms of viral detection and restriction, the influence of host genetic variability on the outcome of disease, and the specifics of antiviral cellular and antibody responses. Much of the research effort is now aimed at understanding what triggers a successful immune response in a minor subset of infected people and how such insights can be harnessed for therapy. This Nature Immunology Focus on the Immunology of HIV (http://www.nature.com/ni/focus/ hiv/index.html) presents five specially commissioned Reviews plus a Perspective that discuss these issues.

Landau and colleagues show how research into accessory proteins expressed by HIV has revealed the ingenious biology of specific host antiviral proteins known as 'restriction factors'. Their Review presents fascinating details of the continuous arms race between viral proteins and their restriction factors, by exploring the interaction among three such pairs, Vif-APOBEC3, Vpx-SAMHD1 and Vpu-tetherin, and offers insight into the mechanisms that dictate, restrict or drive viral evolution during the course of adaptation to new hosts.

Innate effector mechanisms contribute to the control of viremia and modulate the quality of the adaptive immune response. Altfeld and Gale explore the concerted actions of signaling through pathogen-recognition receptors, activation of cells of the innate immune system, cross-talk between the innate immune system and adaptive immune system, and viral evasion strategies that direct the outcome of HIV infection. The authors discuss the biology of two newly characterized sensors of viral reverse transcription, cGAS and IFI16, and discuss how signaling from immunological sensors can induce either cell activation and an interferon-dependent antiviral state or inflammasome activation and death by pyroptosis, depending on the context of the infected cell and the interaction between the virus and host proteins.

Migueles and Connors use insights drawn from the study of people who control HIV infection for long periods in the absence of therapy, known as 'elite controllers' or 'long-term non-progressors', to discuss the features of a $\mathrm{T}$ cell response capable of potent and durable immunological control of HIV-1. The authors summarize arguments indicating that the main controllers of HIV restriction are the $\mathrm{CD} 8^{+} \mathrm{T}$ cells; that differences in the ability to recognize HIV-infected cells and escape mutations are not a barrier for an effective immune response; and that control of viral replication is due to the quantitative features of the $\mathrm{CD}^{+} \mathrm{T}$ cells response-that is, superior ability to produce multiple cytokines and chemokines, proliferate and kill HIV-infected cells. As these are features of an effective cellular response in many chronic infections, understanding the control mechanisms would represent a substantial advance.

Burton and Mascola discuss antibody responses to the viral surface envelope glycoprotein. Antibodies that do not bind to the native envelope trimer, and thus do not neutralize the virus, develop early during infection. Although these antibodies do not put selective pressure on the virus, they can mediate phagocytosis or sequestration of virions. Antibodies that can bind to envelope trimers appear around 3 months after infection, but they neutralize only the infecting HIV strain, and thus the virus can escape their neutralizing activity. Neutralizing antibodies with a broad spectrum of activity against multiple HIV strains take years to evolve, present a level of breadth, potency and functional features not found in most antibody responses, and have great potential for therapy. This Review discusses the structural and immunological features that explain the effectiveness, or lack thereof, of these classes of antibodies.

In a Review looking at the effect of host genetic variation in the outcome of HIV infection, McLaren and Carrington point out that apart from the considerable effect of CCR5, which is the co-receptor for viral entry, and HLA class I, which puts substantial pressure on the virus because of presentation of the viral epitopes, other genetic polymorphisms are probably context dependent. Thus, the positive effects of particular killer immunoglobulin-like receptors are functionally relevant only in the presence of their specific HLA ligands, while genetic variability in a locus that controls the amount of a microRNA that targets the gene encoding an HLA-C molecule is associated with improved control of HIV-1 only in those people with the HLA-C allele (itself polymorphic) with an intact microRNA-binding site. Thus, functional studies and increased insight into the combinatorial traits of the host genetic makeup are essential for understanding the immunological control of HIV.

Finally, in a Perspective, Fauci and colleagues discuss the challenges posed by persistent HIV reservoirs, overview various attempts to purge these reservoirs, such as early initiation of antiretroviral therapy and blockade of immunomodulatory molecules, and propose criteria for establishing attainable goals for HIV therapy.

All the Focus articles point out that multiple lines of evidence, including clinical data from the beginning of the HIV-1 epidemic and subsequent experimental data, suggest that very early events during infection have important consequences for control of this virus. Together with data indicating that the activation of certain pathways of the innate immune system can dictate the strength of the adaptive immune responses, it seems likely that certain desirable characteristics of the innate response to HIV are linked to an efficient adaptive response and viral control. Understanding how the interaction between the host and viral factors contributes to variations in the immune response and disease progression is essential for successful intervention strategies. 